\title{
Surface Innovations: Referees 2021
}

The following is a list of referees who have reviewed papers for Surface Innovations between 1 December 2020 and 30 November 2021.

The Institution of Civil Engineers is very grateful for their assistance.

We are continually looking for suitable reviewers for papers submitted to Surface Innovations. Papers published in ICE Science journals must be submitted to at least two independent referees to judge accuracy, style, impact, importance and interest.

If you are interested in reviewing articles on any topic related to surface innovations, please submit your name, qualifications or CV, and areas of

Lutfi Agartan

Bulent Aktas

Sima Akter

Shariful Amin

Senthil Kumar Arumugam

Sameer Awad

Mahboobeh Azadi

Pradipta Banerjee

Ilker Bayer

Edward Bormashenko

Dmitry Budrevich

Yongwei Cai

Gerardo Callegari

Mirko Černák

Zongjian Chai

Khaled Chemaa

Dexin Chen

Han Chen

Qinjun Chen

Xiaowen Chen

Xian-Wei Cheng

Shu-Lei Chou

Tarun Dam

Yangyang Dang

Thierry Darmanin

Claudio Della Volpe

Öznur Demir Oğuz

Sarwar Ditta

Liubing Dong

Vladimir Dorofeev
Hao Du

Kirill Efimenko

Mai Elkaliuoby

Hossam Emam

Alexandre Emelyanenko

H. Yildirim Erbil

Qingming Feng

Shaofang Fu

Yasser Gaber

Bora Garipcan

Badrudin Gasanov

Gulnara Gaynanova

Hongbo Gu

Sujit Kumar Guchhait

Ahsan Habib

Biao Han

Sadeg Hani

Xiaodong Hong

Ruiqing Hou

Bensheng Huang

Jia-Qi Huang

Hassen Jaafar

Bochen Jiang

Maria Kalinina

Stoyan Karakashev

Saeid Kargozar

Thomas Keller

Kelvii Kwok

Bowen Li

Chilin Li expertise. We are in need of individuals who will agree to review papers in a timely fashion (within 3 to 4 weeks of receipt) and provide confidential feedback to the Editorial Advisory Panel concerning the quality of the paper and any suggested revisions that would be appropriate.

If you are such a person, please contact Rebecca Rivers (tel.: $+44(0)$ 207665 2241; e-mail: rebecca.rivers@ice.org.uk) for more information on the referee process.

Jing Li

Ji Liang

Wenyan Liang

Long Lin

Hao Liu

Mingyan Liu

Shaomin Liu

Zhongkui Liu

Jianmin Ma

Satish Manjare

Muhamed Maqsoud

Abraham Marmur

Raza Miah

Andzej Miszczyk

Masoud Mozafari

Chandra Mohan Singh Negi

Khanali Nekouee

Dinesh Pathak

João Fernando Pereira Gomes

Kenneth Podolak

Anisur Rahman

Mohammad Reza Saeb

Alexis Salazar

Dhamodaran Santhanagopalan

Shahram Shafaei

Koichiro Shiomori

Neil Shirtcliffe

Jia Shuai

Stefano Siboni

Sarabjeet Sidhu
Dong Su

Jiangbin Su

Wan-chang Sun

Ziqi Sun

Harekrushna Sutar

Lilianna Szyk-Warszynska

Katarzyna Szymczyk

Anwar Tozri

Pramanshu Trivedi

Ditta Ungor

Mojtaba Vakili-Azghandi

Jun Wang

Xin-chang Wang

Piotr Warszynski

Pawel Widomski

Xiaogang Yang

Andrey Yaroslavtsev

Junwei Ye

Gulen Yesilbag Tonga

Mehmet Yılmaz

Eui-Sung Yoon

Hongwen Yu

Yakup Yürektürk

Danhui Zhang

Jintao Zhang

Lei Zhang

Xiaomin Zhang

Yao Zheng

Liang Zhou
Yangyang Wen 